\title{
Fear avoidance in CFS/ME: invalid results
}

\author{
Michiel Tack ${ }^{1}$
}

Received: February 7, 2019/Accepted: March 20, 2019/Published online: March 27, 2019

(C) Springer Science+Business Media, LLC, part of Springer Nature 2019

\section{Dear Editor,}

I would like to point out an error in the abstract by Ali et al. (2019). Contrary to what the abstract claims, fear avoidance was not related to fatigue at follow-up (T2). While stronger fear avoidance beliefs were correlated with worse social adjustment at T2 $(p=0.022)$, this was not the case for fatigue severity $(p=0.320)$ or work and school attendance $(p=0.151)$. The authors do not specify how these null results should be interpreted if fear avoidance is indeed an important perpetuating factor in chronic fatigue syndrome/myalgic encephalomyelitis (CFS/ME).

The selection criteria used in this study could also be questioned. In 2015, an NIH report (Green et al., 2015) cautioned that the Oxford definition of CFS/ME "may impair progress and cause harm" as it results in a high risk of including patients with fatiguing illnesses other than CFS/ME. Consequently, research groups tend to use more stringent diagnostic criteria. In the study by Ali et al. however, patients self-reported their diagnosis and $15.7 \%$ did not even meet the Oxford definition. It is therefore unclear if this paper should be seen as a study of CFS/ME, as the title claims. Unexplained chronic fatigue might be a more suitable term.

Contrary to the authors' hypothesis, access to treatment was associated with lower instead of higher work and school attendance. The main treatment in this naturalistic study consisted of graded exercise therapy (GET) and/or cognitive behavioral therapy (CBT). It is possible that some participants deteriorated following GET or CBT, a finding that has been reported in several patient surveys (Kindlon, 2011). As patients were not recruited from clinics, they may have felt less pressure to respond positively to questions about the effects of treatment than if they were returning questionnaires to those who had provided their therapy, which is often the case in clinical trials. This interpretation was not explored by the authors.

Compliance with ethical standards

Conflict of interest The author declare that they have no conflict of interest.

\section{References}

Ali, S., Adamczyk, L., Burgess, M., \& Chalder, T. (2019). Psychological and demographic factors associated with fatigue and social adjustment in young people with severe chronic fatigue syndrome/myalgic encephalomyelitis: A preliminary mixedmethods study. Journal of Behavioral Medicine. https://doi.org/ 10.1007/s10865-019-00010-x

Green, C. R., Cowan, P., Elk, R., O’Neil, K. M., \& Rasmussen, A. L. (2015). National institutes of health pathways to prevention workshop: Advancing the research on myalgic encephalomyelitis/chronic fatigue syndrome. Annals of Internal Medicine, 162, 860-868.

Kindlon, T. (2011). Reporting of harms associated with graded exercise therapy and cognitive behavioural therapy in myalgic encephalomyelitis/chronic fatigue syndrome. Bulletin of the IACFS/ME, 19, 59-111.

Publisher's Note Springer Nature remains neutral with regard to jurisdictional claims in published maps and institutional affiliations.

Michiel Tack

tackmichiel@gmail.com

1 Oudenaarde, Belgium 